\title{
Superior vena caval stenosis: a complication of transvenous endocardial pacing
}

\author{
D M MATTHEWS ${ }^{1}$ AND J C FORFAR
}

From the Department of Cardiology, Royal Infirmary, Edinburgh, UK

Superior vena caval obstruction is a rare complication of transvenous endocardial pacing and is usually the result of thrombus formation round the pacing electrode (Kosowsky and Barr, 1972). We report a case of superior vena caval obstruction without thrombus formation secondary to localised stenosis at the site of the proximal cut end of a retracted endocardial electrode. This complication of transvenous pacing electrodes has not been described previously.

\section{Case report}

A 60-year-old woman presented in December 1971 with three syncopal episodes typical of Stokes-Adams attacks. A permanent Devices demand endocardial ventricular pacemaker was inserted via the left cephalic vein with the generator in a left infraclavicular subcutaneous pocket.

Over the next year she had sudden onset of right hemiparesis on two occasions, each with full recovery apart from residual expressive dysphasia. Long-term warfarin treatment was maintained thereafter for presumed cerebral emboli (the prothrombin ratio being maintained within the therapeutic range on most occasions). Transient right-sided focal fits developed in 1973 and were successfully managed with phenytoin. The pacemaker box was changed at 28 months because of battery failure, and a Vitatron demand unit was attached to the original electrode. Eighteen months later a discharging wound sinus necessitated removal of the Vitatron box and its replacement with an epicardial system. The endocardial wire could not be detached from the apex of the right ventricle and the electrode was, therefore, cut distally in the infraclavicular wound and left in situ.

In July 1978 she was admitted with a two-week history of progressive swelling of the face, neck, and arms. There was clinical evidence of obstruction of the superior vena cava. Chest radiograph showed no evidence of extrinsic caval compression. A superior venocavogram showed a tight stenosis (fig 1) at the upper end of the SVC at the site of retraction of the retained endocardial lead. There was no reflux of dye into the left innominate vein, which was interpreted as due to thrombotic occlusion of this vessel.

The patient was treated with intravenous heparin and diuretics, but her condition deteriorated progressively with increasing drowsiness and eventual coma. She died eight days after admission.

At necropsy there was evidence of recent bilateral ${ }^{1}$ Present address : Deaconess Hospital, 148 The Pleasance, Edinburgh.

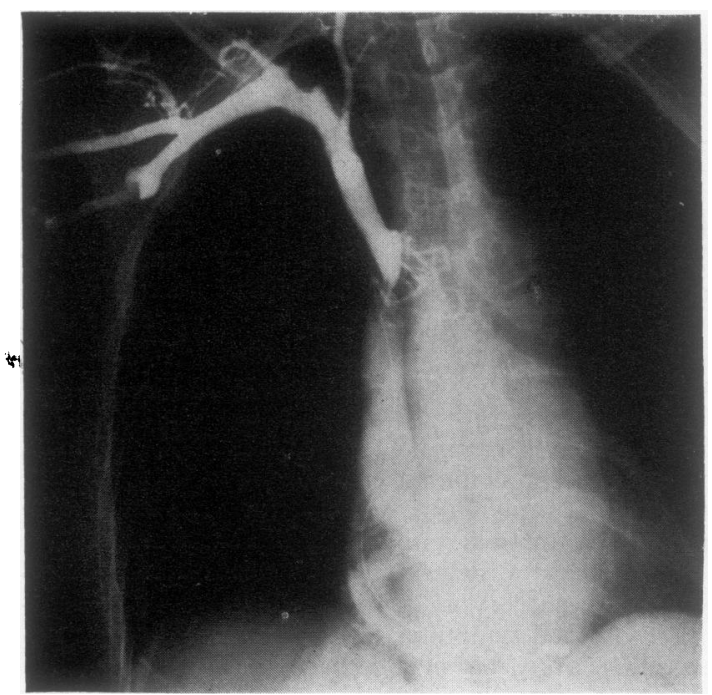

Fig 1 Cavogram showing stenosis of SVC at innominate junction with collateral vein formation and endocardial electrode buried in $S V C$ wall.

subdural haemorrhage. There was no evidence of thrombus formation in SVC, innominate, or jugular veins. The coiled proximal end of the retained endocardial wire was buried in a tight fibrotic stenosis at 3 the origin of the SVC (fig 2). The distal tip of the electrode was epithelialised with only a small amount of thrombus at the site of ventricular attachment. There was no thrombus within the atria or major pul- 음 monary vessels. The foramen ovale was not patent.

\section{Discussion}

Complications of endocardial pacing elect clude cardiac perforation, electrode displacement, in- 0 fection, and venous thrombosis and embolism $\omega$ (Kosowsky and Barr, 1972). In previous descriptions of caval occlusion the cause has been thrombus roundo a normally functioning or retained endocardial lead $\underset{\mathbb{D}}{\overparen{D}}$ (Wertheimer et al, 1973; Williams and Demos, 1974; $\stackrel{\mathcal{O}}{+}$ Chamorro et al, 1978). Major pulmonary embolism 0 from this source has also been described (Kaulbach $\overline{0}$ and Krukonis, 1970).

Our patient was treated with heparin and diuretics $\frac{\rho}{\Phi}$ in the belief that thrombus was the cause of the caval 2 obstruction and in the knowledge that such manage- 


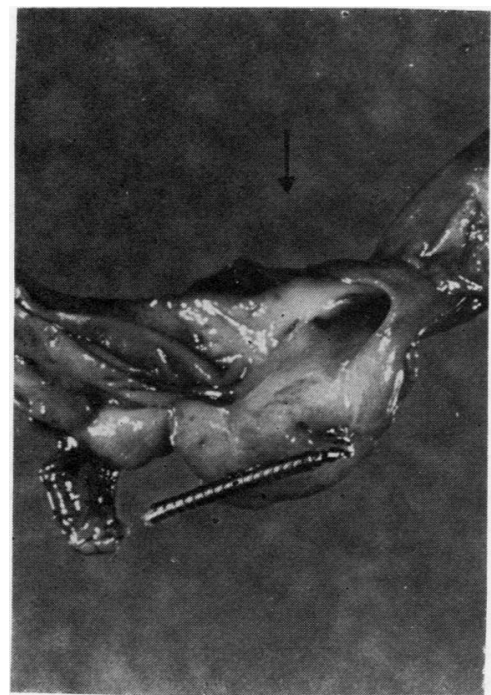

Fig 2 Fibrotic stenosis of SVC at proximal end of retained endocardial wire.

ment had been successful in similar cases previously (Wertheimer et al, 1973; Chamorro et al, 1978). Streptokinase (Williams and Demos, 1974) was not used because of the concurrent administration of warfarin. Anticoagulant treatment was probably not beneficial and may have contributed to death from subdural haemorrhage in the presence of cerebral venous hypertension.

In retrospect the treatment of choice for this patient would have been removal of the retained endocardial wire and resection or bypass of the area of stenosis. This approach has been used successfully in the management of caval obstruction complicating ventriculoatrial shunts (Overton et al, 1966). The dangers of a retained endocardial wire are not insignificant, and every effort should be made to remove them if no longer required. If this is not possible the proximal end of the electrode should be firmly attached under the skin to prevent its retraction to a major vein.

We thank Dr R M Marquis for guidance in preparing this report, Dr T A S Buist for performing the cavogram, and Dr D Thompson for his necropsy.

\section{References}

Chamorro, H, Rao, G, and Wholey, M H (1978). Superior vena caval syndrome: a complication of transvenous endocardial cardiac pacing. Radiology, 126, 377-378.

Kaulbach, M G, and Krukonis, E E (1970). Pacemaker electrode-induced thrombosis in the superior vena cava with pulmonary embolism-a complication of pervenous pacing. American Journal of Cardiology, 26, 205-207.

Kosowsky, B D, and Barr, I (1972). Complications and malfunctions of electrical cardiac pacemakers. Progress in Cardiovascular Diseases, 14, 501-513.

Overton, M C (III), Derrick, J, and Snodgrass, S R (1966). Surgical management of superior vena cava obstruction complicating ventriculoatrial shunts. Journal of Neurosurgery, 25, 164-171.

Wertheimer, M, Hughes, R K, and Hilmon-Castle, C (1973). Superior vena caval syndrome: complication of permanent transvenous endocardial cardiac pacing. Journal of the American Medical Association, 224, 1172-1173.

Williams, D R, and Demos, N J (1974). Thrombosis of superior vena cava caused by pacemaker wire and managed with streptokinase. Journal of Thoracic and Cardiovascular Surgery, 68, 134-136.

Requests for reprints to: Dr J C Forfar, Department of Cardiology, Royal Infirmary, Lauriston Place, Edinburgh EH3 9YW. 